\title{
In-Line Chromatic Dispersion Measurement for NRZ and RZ Signals Using a Novel RF Spectrum Phase Detection Technique
}

\author{
Guozhou Jiang, Ying Mei \\ College of Educational Information \& Technology, Hubei Normal University, Huangshi, China \\ Email:jgz_hust@163.com
}

Received May 17, 2013; revised June 20, 2013; accepted July 23, 2013

Copyright (C) 2013 Guozhou Jiang, Ying Mei. This is an open access article distributed under the Creative Commons Attribution License, which permits unrestricted use, distribution, and reproduction in any medium, provided the original work is properly cited.

\begin{abstract}
In this paper, a novel method of in-line chromatic dispersion (CD) measurement is proposed, we theoretically and experimentally demonstrated the CD measurement of $10-\mathrm{Gbit} / \mathrm{s}$ NRZ and RZ signals by a novel RF spectrum phase detection technique, this is performed using in-band tone monitoring RF, electrically down-converted to direct current (DC) or a low intermediate-frequency (IF) of less than $1 \mathrm{MHz}$ through electronic mixing with local oscillator (LO) of $2.4 \mathrm{GHz}$. The measurement provides a large CD measuring range with good accuracies $( \pm 2000 \pm 35 \mathrm{ps} / \mathrm{nm})$, and independent of the bit-rate and data format. In addition, the use of electronic mixer and low-speed detectors makes it cost effective for in-line CD measurement.
\end{abstract}

Keywords: Radio Frequency Photonics; Frequency Modulation; Fiber Measurements; Fiber Optics Links; Subsystems; Modulation; Ultrafast Processes in Fibers

\section{Introduction}

With the increased demand for large data capacity, higher data rates have become an essential requirement of next generation light-wave system. It has been recognized that at data rates higher than $10 \mathrm{~Gb} / \mathrm{s}$, it will be necessary to provide tunable dispersion compensators (TDC) in order to accommodate uncertainties and dynamic chromatic dispersion (CD) variations in link, various tunable dispersion compensators have been demonstrated [1]. For such devices to be working effectively, an appropriate residual CD measuring and feedback signal must be obtained from the data stream passing through the TDC. Various feedback signals are possible including, bit-error rate (BER) derived from forward error correction algorithms, eye monitoring e.g [2], and schemes that rely on monitoring the RF spectrum of the detected bit stream after a fast photo-detector e.g [3], but these feedback signals can't indicate the value of residual $\mathrm{CD}$, and it is necessary to adjust TDC till obtain a perfect state of feedback signals, that need a lot of time for adjusting, if residual $\mathrm{CD}$ value changed, the process must be iterated.

So some researchers have focused on researching of residual CD monitoring, and there have been several ap- proaches of CD monitoring recently demonstrated, including asynchronous sampling and histogram evaluation [4-8], electrical dispersion equalizer [9], self-phase modulation, four-wave mixing, and cross-phase modulation (XPM) in optical fibers [10-12], radio-frequency (RF) tone measurement $[13,14]$. However, some of these approaches tends to require either high-speed components (e.g., oscilloscope, detector, RF spectrum analyzer, or analog-to-digital converter), a tunable DLI to decode phase information into amplitudes, or high data input power.

\section{Principle of CD Measurement}

The model of this technique is depicted in Figure 1. This technique is based on electrical mixing with orthogonal I-Q procedure.

The incoming signal $E_{D S B}(t)$ is a modulated dual sideband (DSB) signal, two single sideband (SSB) tunable band-pass optical filter is used to distill upper and lower sideband of the incoming signal, that the upper sideband signal and lower sideband signal are respectively given by:

$$
E_{L}(t)=\alpha \sqrt{I_{0}} \cos \left(\omega_{0} t\right)+\beta \sqrt{I_{0}} \cos \left(\left(\omega_{0}-\omega_{d}\right) t+\varphi_{L}\right),(1)
$$




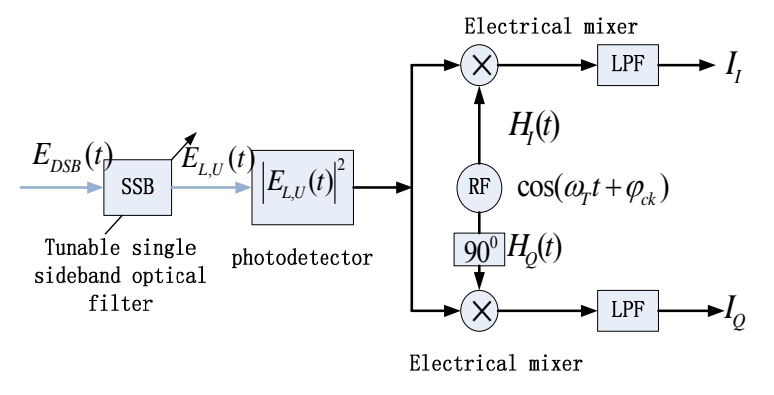

Figure 1. Principle of CD measurement based on RF spectrum phase detection using electrical mixing.

$$
E_{U}(t)=\alpha \sqrt{I_{0}} \cos \left(\omega_{0} t\right)+\beta \sqrt{I_{0}} \cos \left(\left(\omega_{0}+\omega_{d}\right) t+\varphi_{U}\right) .
$$

After the photo-detector and the electrical mixing, the in-phase and in-quadrature received signals $I_{I, Q}$ for the LSB and USB are given by:

$$
\begin{gathered}
I_{I U}=\left|E_{U}(t)\right|^{2} \times H_{I}(t), \\
I_{Q U}=\left|E_{U}(t)\right|^{2} \times H_{Q}(t), \\
I_{I L}=\left|E_{L}(t)\right|^{2} \times H_{I}(t), \\
I_{Q L}=\left|E_{L}(t)\right|^{2} \times H_{Q}(t),
\end{gathered}
$$

where $H_{I}(t)$ and $H_{Q}(t)$ are the electrical mixer transfer functions for the in-phase and in-quadrature signals $I_{I, Q}$, respectively given by:

$$
\begin{gathered}
H_{I}(t)=\cos \left(\omega_{T} t+\varphi_{c k}\right), \\
H_{Q}(t)=\cos \left(\omega_{T} t+\varphi_{c k}+\frac{\pi}{2}\right)
\end{gathered}
$$

where $\omega_{T}$ is the RF modulation frequency of the LO signal and the $\varphi_{c k}$ is its relative phase to the data clock.

When $\omega_{T}=\omega_{d}$, the detected signals $I_{I U}, I_{I L}, I_{Q L}$, $I_{\mathrm{QU}}$ after low pass filter (LPF) are determined from Equations (9)-(12)

$$
\begin{array}{r}
I_{I L}=\frac{\alpha \beta I_{0}}{2} \cos \left(\varphi_{L}+\varphi_{c k}\right), \\
I_{Q L}=\frac{\alpha \beta I_{0}}{2} \cos \left(\varphi_{L}+\varphi_{c k}+\frac{\pi}{2}\right), \\
I_{I U}=\frac{\alpha \beta I_{0}}{2} \cos \left(\varphi_{U}+\varphi_{c k}\right), \\
I_{Q U}=\frac{\alpha \beta I_{0}}{2} \cos \left(\varphi_{U}+\varphi_{c k}+\frac{\pi}{2}\right),
\end{array}
$$

The relative phase of the carrier and each sideband for the LSB and USB ( $\varphi_{L}$ and $\left.\varphi_{U}\right)$ are given by:

$$
\varphi_{L}=\operatorname{Arctg}\left(\frac{I_{Q L}}{I_{I L}}\right)-\varphi_{c k},
$$

$$
\varphi_{U}=\operatorname{Arctg}\left(\frac{I_{Q U}}{I_{I U}}\right)-\varphi_{c k} .
$$

It is noted that the phase difference $\Delta \varphi$ is independent of LO signal's phase $\varphi_{c k}$, and $\Delta \varphi$ is given by:

$$
\Delta \varphi=\varphi_{U}-\varphi_{L}=\operatorname{Arctg}\left(\frac{I_{Q U}}{I_{I U}}\right)-\operatorname{Arctg}\left(\frac{I_{Q L}}{I_{I L}}\right) .
$$

The GVD is then given by:

$$
G V D=\frac{2 \pi c \Delta \varphi}{\lambda^{2} \omega_{T}^{2}},
$$

\section{Experimental Implementation}

Experimental demonstration was performed using the setup of Figure 2. The transmitter comprises a tunable laser (TL) operating at $1550 \mathrm{~nm}$ with $10 \mathrm{MHz}$ line width, the part of PRBS generating $10 \mathrm{Gbit} / \mathrm{s}$ pseudorandom bit sequence (PRBS), and pulse generator generats 66\% NRZ and RZ pulse shape respectively, the optical carrier was modulated with a $10 \mathrm{GHz}$ NRZ/RZ PRBS of length $2^{15}-1$ through Mach-Zenhder modulator (MZM). The fiber under test (FUT) comprises single mode fiber (G.652) and dispersion compensation fiber. An erbium-doped fiber amplifier (EDFA) was used to compensate the fiber loss. At the receiver, a tunable optical band-pass filter with $3 \mathrm{~dB}$ bandwidth of $0.6 \mathrm{~nm}$ was used to eliminate the redundant amplified spontaneous emission (ASE) noise, the Mach-Zenhder interferometer (MZI) with FSR (Free Spectrum Range) of $20 \mathrm{GHz}$ was used to distill upper and lower signal band, the optical signal of upper and lower band were respectively detected by analog detector with $3 \mathrm{~dB}$ bandwidth of $3 \mathrm{GHz}$, the output RF electrical signal was split into I and Q channels for mixing with local oscillator (LO), and I channel LO signal has $\pi / 2$ phase difference with $\mathrm{Q}$ channel LO signal. In this experiment we used ADL5382 to actualize mixing RF with LO signal, the LO frequency is $2.4 \mathrm{GHz}$. The analogdigital-converters (ADC) with 14

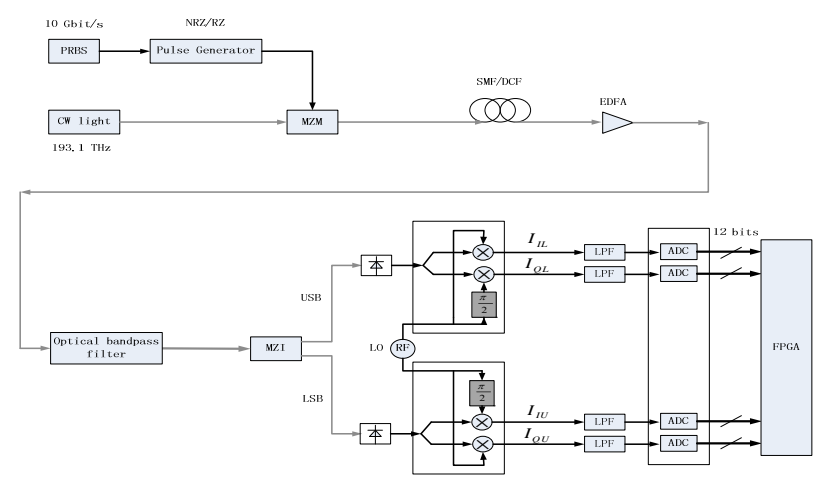

Figure 2. Experimental setup of CD measurement for NRZ and RZ systems. 
bit-width and $20 \mathrm{MHz}$ sample rate were used to sampling the output analog intermediate frequency (IF) signals and converting to digital signals, signals processing was performed in Field Programmable Gate Array (Xilinx: XC4VLX15).

\section{Experimental Results}

The positive chromatic dispersion was added to the signal using five spools of SMF of 20,40,60,80, $100 \mathrm{~km}$ corresponding, respectively, to 335, 670, 1005, 1340 and $1675 \mathrm{ps} / \mathrm{nm}$. The negative chromatic dispersion was added to the signal using four spools of DCF of 3, 6, 9, 12 $\mathrm{km}$ corresponding, respectively, to $-420,-840,-1260$, $-1680 \mathrm{ps} / \mathrm{nm}$. The OSNR was varied with a variable noise loading stage using an ASE source. The OSNR was maintained at a $20.5 \mathrm{~dB}$ level for all $\mathrm{CD}$ measurements. The $\mathrm{CD}$ measurement was tested without introducing any DGD. Figure 3 shows the experimental results of the NRZ format signal. Figure 4 shows the experimental results of the RZ format signal.

The precision of the dispersion measurement mainly depends on the OSNR. Figures 5(a) and (b) shows the detected phase corresponding to different OSNR under the $\mathrm{CD}$ of $0 \mathrm{ps} / \mathrm{nm}$. In measurement, the result of NRZ link is more precise than RZ link under the same OSNR.

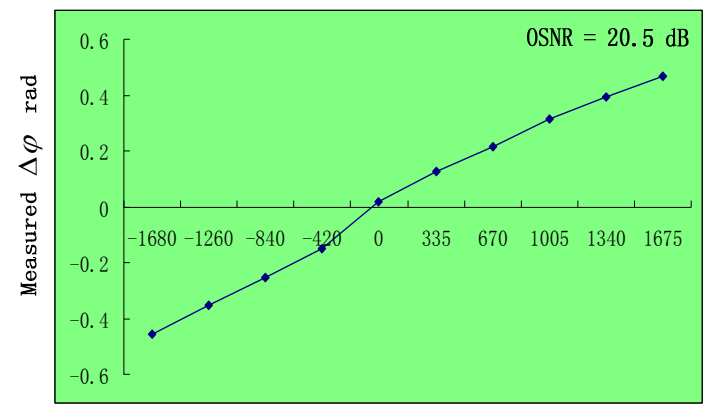

Actual CD ps/nm

(a)

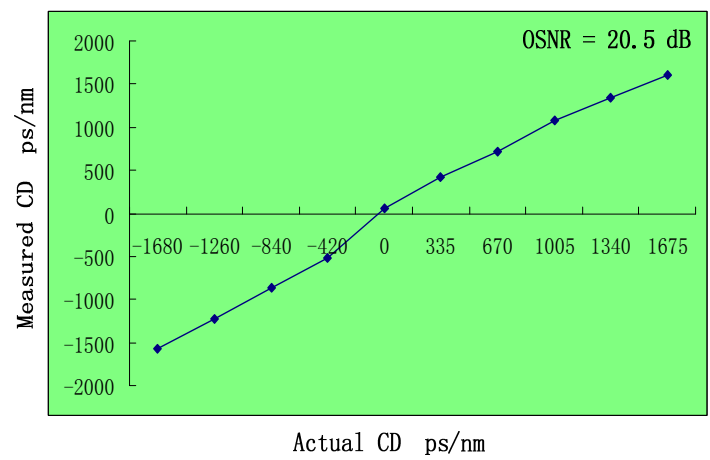

(b)

Figure 3. (a) Measured value of $\Delta \varphi$ corresponding to variable CD (LO = $2.4 \mathrm{GHz}$ ); (b) Measured CD corresponding to actual CD $(\mathrm{LO}=2.4 \mathrm{GHz})$.

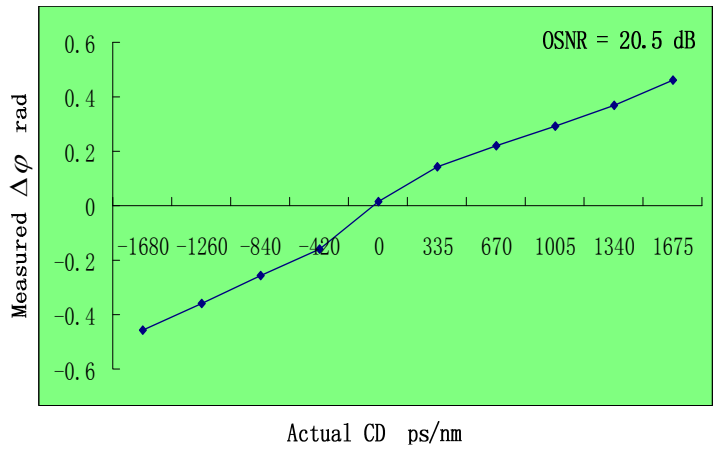

(a)

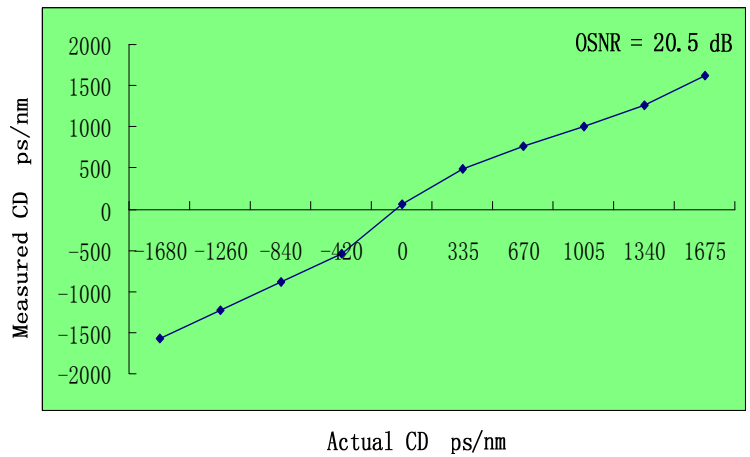

(b)

Figure 4. (a) Measured value of $\Delta \varphi$ corresponding to variable CD (LO = $2.4 \mathrm{GHz}$ ); (b) Measured CD corresponding to actual CD (LO = 2.4 GHz).

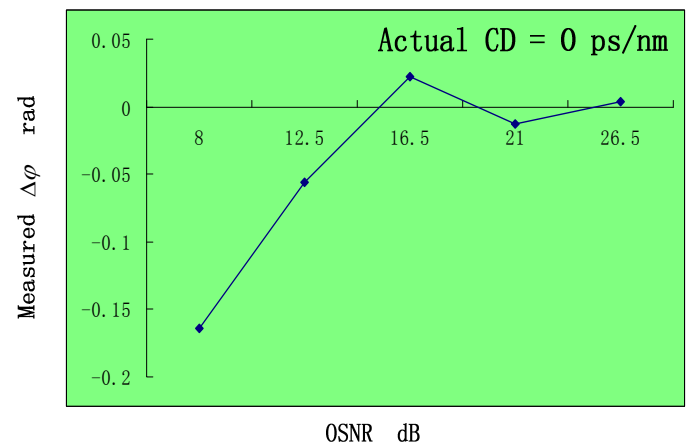

(a)

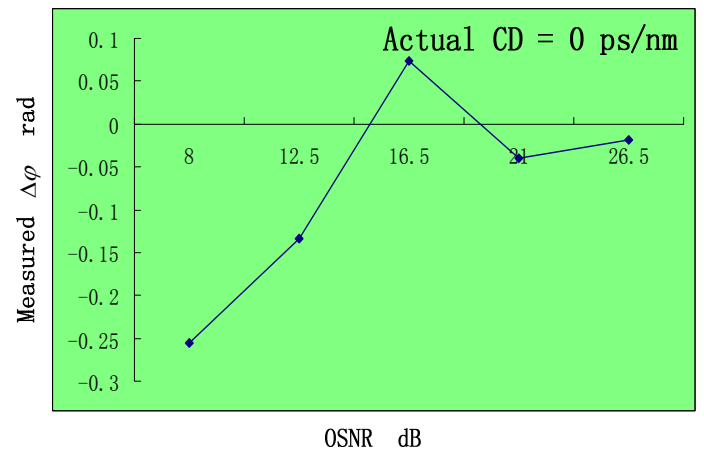

(b)

Figure 5. Detected phase corresponding to different OSNR for 10 G, (a) NRZ; (b) RZ. 


\section{Conclusion}

In this experiment, $\mathrm{CD}$ measuring technique for independent of data rates and formats has been developed, the performance of the technique was experimentally and respectively assessed for $10 \mathrm{Gbit} / \mathrm{s} \mathrm{NRZ}$ and RZ systems. The measurement range and error achieved with the proposed method, based on monitoring a $2.4 \mathrm{GHz}$ inband tone, was $\pm 2000 \pm 35 \mathrm{ps} / \mathrm{nm}$ for CD. In condition of $2.4 \mathrm{GHz} \mathrm{LO}$, the maximum measurement range may up to $\pm 9000 \mathrm{ps} / \mathrm{nm}$, the $\mathrm{LO}$ is tunable that adjust it up for $\mathrm{CD}$ range. The technique was shown to operate in single wavelength system, it can also be used in WDM system by using a tunable optical filter (TOF) to achieve multiwavelength channels $\mathrm{CD}$ measurement in serial mode, or by using array waveguide gates (AWG) to achieve all wavelength channels $C D$ measurement in parallel mode. The minimum acquisition-time is found to be a trade-off between the required measurement accuracy and the monitoring speed. The $5 \mathrm{~ms}$ acquisition time, required to obtain the accuracy stated above, is suitable for measuring application in dynamic optical networks with reconfiguration times greater than that, such as ASON, OBS or OPS systems. Detuning of the MZI optical filter from the optimum position by up to $5 \mathrm{GHz}$ does not have any effect on the phase measurement for the $\mathrm{CD}$ measuring. In addition, the use of electronic mixer for spectra downconversion and the use of low-speed detectors make it potentially cost effective for multi-channel operation.

\section{REFERENCES}

[1] B. J. Eggleton, A. K. Ahuja, P. S. Westbrook, J. A. Rogers, P. Kuo, T. N. Nielsen and B. Mikkelsen, "Integrated Tunable Fiber Gratings for Dispersion Management in High-Bit Rate Systems," Journal of Lightwave Technology, Vol. 18, No. 10, 2000, pp. 1418-1432. doi:10.1109/50.887194

[2] N. Liu, W. D. Zhong, Y. J. Wen and Z. H. Li, "New Transmitter Configuration for Subcarrier Multiplexed DPSK Systems and Its Applications to Chromatic Dispersion Monitoring," Optics Express, Vol. 15, No. 3, 2007, pp. 839-844. doi:10.1364/OE.15.000839

[3] H. Ohta, S. Nogiwa, Y. Kawaguchi and Y. Endo, "Measurement of $200 \mathrm{Gbit} / \mathrm{s}$ Optical Eye Diagram by Optical Sampling with Gain-Switched Optical Pulse," Electronics Letters, Vol. 36, No. 8, 2000, pp. 737-739. doi:10.1049/el:20000538

[4] Z. Q. Pan, Q. Yu, Y. Xie, S. A. Havstad, A. E. Willner, D. S. Starodubov and J. Feinberg, "Chromatic Dispersion Monitoring and Automated Compensation for NRZ and RZ Data Using Clock Regeneration and Fading without Adding Signaling," Optical Fiber Communication Con- ference and Exhibit, Anaheim, 17-22 March 2001, Article ID: WH5-1-3.

[5] S. D. Dods, T. B. Anderson, K. Clarke, M. Bakaul and A. Kowalczyk, "Asynchronous Sampling for Optical Performance Monitoring," Optical Society of America (OFC). Anaheim, 25-29 March 2007, pp. OMM5-1-3.

[6] B. Kozicki, A. Maruta and K. I. Kitayama, "Transparent Performance Monitoring of RZ-DQPSK Systems Employing Delay-Tap Sampling," Journal of Optical Networking, Vol. 6, No. 11, 2007, pp. 1257-1269. doi:10.1364/JON.6.001257

[7] B. Kozicki, A. Maruta and K. I. Kitayama, "Experimental Demonstration of Optical Performance Monitoring for RZ-DPSK Signals Using Delay-Tap Sampling Method," Optics Express, Vol. 16, No. 6, 2008, pp. 3566-3576. doi:10.1364/OE.16.003566

[8] Z. H. Li and G. F. Li, "Chromatic Dispersion and Polarization-Mode Dispersion Monitoring for RZ-DPSK Signals Based on Asynchronous Amplitude-Histogram Evaluation," Journal of Lightwave Technology, Vol. 24, No. 7, 2006, pp. 2859-2866. doi:10.1109/JLT.2006.876089

[9] Z. H. Li, Z. Jian, L. H. Cheng, Y. F. Yang, C. Lu, A. P. T. Lau, C. Y. Yu, H. Y. Tam and P. K. A. Wai, "Signed Chromatic Dispersion Monitoring of $100 \mathrm{Gbit} / \mathrm{s}$ CS-RZ DQPSK Signal by Evaluating the Asymmetry Ratio of Delay Tap Sampling," Optics Express, Vol. 18, No. 3, 2010, pp. 3149-3157. doi:10.1364/OE.18.003149

[10] W. Chen, F. Buchali, X. W. Yi, W. Shieh, J. S. Evans and R. S. Tucker, "Chromatic Dispersion and PMD Mitigation at $10 \mathrm{~Gb} / \mathrm{s}$ Using Viterbi Equalization for DPSK and DQPSK Modulation Formats," Optics Express, Vol. 15, No. 9, 2007, pp. 5271-5276. doi:10.1364/OE.15.005271

[11] P. S. Westbrook, B. J. Eggleton, G. Raybon, S. Hunsche, T. H. Her, "Measurement of Residual Chromatic Dispersion of a $40-\mathrm{Gb} / \mathrm{s}$ RZ Signal via Spectral Broadening," IEEE Photonics Technology Letters, Vol. 14, No. 3, 2002, pp. 346-348. doi:10.1109/68.986808

[12] S. P. Li and D. V. Kuksenkov, "A novel dispersion monitoring technique based on four-wave mixing in optical fiber," IEEE Photonics Technology Letters, Vol. 16, No. 3, 2004, pp. 942-944. doi:10.1109/LPT.2004.823751

[13] T. Luo, C. Y. Yu, Z. Q. Pan, Y. Wang, J. E. McGeehan, M. Adler and A. E.Willner, "All-Optical Chromatic Dispersion Monitoring of a 40-Gb/s RZ Signal by Measuring the XPM-Generated Optical Tone Power in a Highly Nonlinear Fiber," IEEE Photonics Technology Letters, Vol. 18, No. 2, 2006, pp. 430-432. doi:10.1109/LPT.2005.862359

[14] Y. K. Lizé, L. C. Christen, J. Y. Yang, P. Saghari, S. R. Nuccio, A. E. Willner and R. Kashyap, "Independent and Simultaneous Monitoring of Chromatic and Polarization-Mode Dispersion in OOK and DPSK Transmission," IEEE Photonics Technology Letters, Vol. 19, No. 1, 2007, pp. 3-5. doi:10.1109/LPT.2006.888039 